\title{
Association of parental investment of time and cost in food provisioning with adherence to dietary guidelines for the consumption of fruits, vegetables and non-core foods in pre-schoolers
}

\author{
Si Xian Jasmine Lee ${ }^{1,2}$, Tracy Burrows ${ }^{1,2,3}$, Serene Yoong ${ }^{1,2,4}$ and Rebecca Wyse ${ }^{1,2,4, *}$ \\ ${ }^{1}$ Faculty of Health and Medicine, University of Newcastle, University Drive, Callaghan, NSW 2308, Australia: \\ ${ }^{2}$ Hunter Medical Research Institute (HMRI), Newcastle, NSW, Australia: ${ }^{3}$ Priority Research Centre for Physical \\ Activity and Nutrition, University of Newcastle, Callaghan, NSW, Australia: ${ }^{4}$ Hunter New England Population \\ Health, Hunter New England Local Health District, Wallsend, NSW, Australia
}

Submitted 22 September 2017: Final revision received 3 April 2018: Accepted 8 May 2018: First published online 1 June 2018

\begin{abstract}
Objective: To assess whether parent-reported time and cost for provision of food is associated with consumption of fruits, vegetables and non-core foods in preschoolers.

Design: Cross-sectional analysis of baseline data from the Healthy Habits randomised controlled trial. Two subscales of the Children's Dietary Questionnaire (CDQ) were used to assess fruit and vegetable, and non-core food consumption. Setting: Thirty pre-schools in the Hunter Region of New South Wales, Australia. Subjects: Parents ( $n$ 396) with a child aged $3-5$ years attending a participating pre-school were recruited. Parents needed to reside with that child for at least four days per week and have primary responsibility for providing meals and snacks to their child.

Results: Sixty-three per cent of children had a parent-reported subscale score indicating adherence to dietary guidelines for fruit and vegetable intake, while $64 \%$ of children had a subscale score indicating they were exceeding dietary guidelines for non-core foods. Regression models revealed significant positive associations between higher CDQ scores for non-core foods (indicating higher consumption levels) and minutes that parents spent preparing food $(P=0.032$ and $0.025)$ and amount spent on purchasing food $(P=0.043$ and 0.020$)$. The magnitude of the effects was small (estimate $=0.003$ and 0.001).

Conclusions: Time and cost spent by parents on provision of food was not significantly associated with child fruit and vegetable consumption. Further explorations into time spent on food preparation and cost of food procurement are warranted to help address the increased consumption of non-core foods by pre-school children.
\end{abstract}

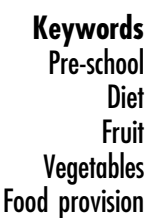

Keywords

Diet

Fruit

Food provision
The burden of disease from inadequate consumption of fruits and vegetables is a global public health concern, accounting for $10 \%$ of the global disease burden and 6.7 million associated deaths in 2010, with substantial personal, community and economic costs ${ }^{(1-4)}$. Higher fruit and vegetable intake is associated with decreased risk of child and adult obesity and CVD ${ }^{(5,6)}$. Internationally, children in developed countries consume insufficient fruits and vegetables to meet the WHO guideline of at least $400 \mathrm{~g}$ of fruits and vegetables combined per day ${ }^{(7)}$. In Australia, dietary guidelines recommend that 4-8-year-olds consume
1.5 servings of fruits and 4.5 servings of vegetables daily ${ }^{(8)}$ However, only $5 \cdot 1 \%$ of children and adolescents (aged 2-18 years) are meeting the national recommended guidelines for both fruit and vegetable intake ${ }^{(9)}$. The consumption of adequate amounts of fruits and vegetables by children is essential as it allows for the provision of vital nutrients necessary for healthy growth and development ${ }^{(10)}$.

Energy-dense, nutrient-poor foods are categorised as 'discretionary' foods in the Australian Dietary Guidelines (also known as 'non-core') and are typically high in fat, 
sugar and/or salt ${ }^{(11)}$. Australian guidelines recommend 4-8-year-olds consume no more than 2.5 servings daily. The excessive consumption of such foods is linked to the development of childhood overweight and obesity, diabetes, CVD and cancer ${ }^{(12,13)}$. In the 2007 Australian National Child Nutrition and Physical Activity Survey, children aged 2-7 years were reported to consume 33$35 \%$ of their daily energy intake from non-core foods ${ }^{(14)}$ despite recommendations that these foods should account for only $5-20 \%{ }^{(15)}$. Given that food habits established in childhood track into adulthood $^{(16)}$ and are associated with the risk of developing chronic disease, introducing interventions to increase consumption of fruits and vegetables and decrease consumption of non-core foods in childhood may have the potential to reduce morbidity and associated burden in adulthood ${ }^{(17)}$.

During childhood there is reliance on parents and carers for the provision of children's food ${ }^{(18)}$, with previous research reporting a positive association between children's consumption of fruits and vegetables and the extent to which parents provide children with these foods ${ }^{(19,20)}$. The provision of food process is a cycle recognised in the literature and comprises five steps, including (i) acquisition, (ii) preparation and (iii) cooking, all of which are precursors to (iv) eating and (v) disposal ${ }^{(21)}$. Any disruption or barriers in the three steps of acquisition, preparation and cooking can potentially disrupt or affect food consumption through influencing the availability and access to food items. The literature has previously identified a number of barriers faced by parents in the process of food provision for their children ${ }^{(21)}$. Common barriers to the provision of healthy foods include the time and costs involved in food acquisition, preparation and cooking ${ }^{(6,22-24)}$.

A 2015 systematic review of obesogenic dietary factors of children aged 0-6 years identified that parents perceive healthy foods as more expensive than unhealthy foods ${ }^{(6)}$. A number of qualitative studies with parents of children of pre-school age similarly report that parents perceive time and cost issues as key constraints to provision of healthier foods for their children ${ }^{(6,22,25)}$. Furthermore, a crosssectional study of 550 parents and children (aged 6-11 years) found lower child consumption of fruits and vegetables in families with the lowest household grocery expenditure relative to those with the highest grocery expenditure $^{(26)}$. The issue of affordability of healthier foods might be more substantial for those in remote locations $^{(27)}$. In addition, the issue of perceived cost may lead to some parents buying increased amounts of cheaper convenience foods despite being aware of the benefits of healthy eating ${ }^{(22)}$.

Time scarcity is also an issue of increasing salience as more parents join the workforce ${ }^{(28)}$, reducing the time available for the preparing and cooking steps within the provisioning process and increasing reliance on eating out and convenience foods (e.g. fast-food outlets) to save time ${ }^{(6,22)}$. A survey of 600 American adults in 2014 reported that participants perceived purchasing and preparing of fruits (18\%) and vegetables (22\%) to be timeconsuming ${ }^{(29)}$. Meanwhile, cross-sectional studies of American and New Zealand adults suggest that more time spent on home food preparation is associated with higher consumption of fruits and vegetables and lower consumption of non-core foods ${ }^{(21,30-32)}$.

The literature to date regarding the impact of time and cost on healthier food consumption has primarily been focused on the dietary intake of adults, with few studies assessing associations between time and cost of food procurement and dietary intake in young children. The Healthy Habits Trial was a cluster-randomised controlled trial testing the efficacy of a telephone-based intervention targeting parents to increase the fruit and vegetable consumption of their pre-school children (3-5 years) ${ }^{(33)}$. Almost 400 parents were recruited to this trial, providing an opportunity to address additional identified gaps in the literature $^{(34)}$. Therefore, using baseline data collected from the Healthy Habits Trial, the aim of the present study was to assess whether parent-reported time and cost to procure and prepare foods is associated with the consumption of fruits, vegetables and non-core foods in children of pre-school age (i.e. 3-5 years old). It was hypothesised that more investment in time and cost of food preparation would be associated with higher fruit and vegetable intake and lower non-core food intake.

\section{Methods}

\section{Study design}

The study design was cross-sectional and utilised baseline data collected from the Healthy Habits cluster-randomised controlled trial. The methods herein describe the relevant processes for collecting baseline data as part of the Healthy Habits Trial ${ }^{(33)}$.

\section{Ethical approval}

Study procedures were approved by the Hunter New England Human Research Ethics Committee (reference number 08/10/15/5.09) and the University of Newcastle Human Research Ethics Committee. Written informed consent to participate in the Healthy Habits Trial was obtained from all parents.

\section{Participants and recruitment}

Parents were recruited from thirty non-government preschools (i.e. private) in the Hunter Region of New South Wales (NSW), Australia, in 2010. These non-Department of Education schools comprise the majority of pre-schools in the Hunter Region ( $90 \%)$. A list of pre-schools in the Hunter Region ( $n$ 57) was obtained from a database maintained by the NSW Department of Community 
Services (the licensing agency for the sector) and assessed by researchers for eligibility. Pre-schools were ineligible ( $n$ 19) if they: provided any meals to children; catered exclusively to children with special needs (children who require specialist care); or had participated in any child healthy eating research projects within the previous six months. Supervisors from eligible pre-schools were mailed study information and consent forms. Approximately two weeks later, the supervisors received a telephone call from a research assistant who answered any questions they may have had, confirmed pre-school eligibility and prompted supervisors to return the consent form.

Supervisors at consenting pre-schools were given flyers to distribute to parents to encourage recruitment. In most cases, a research assistant was on site at the pre-school for a minimum of one hour per day during either parent drop off or pick up, every day for one week, to answer any enquiries about the research and distribute the recruitment packs to parents. Recruitment packs for parents contained an information sheet, a consent form and a return envelope. Two weeks following the dissemination of the recruitment packs, parents were given reminder letters by the pre-schools to encourage the return of their consent forms. Consent forms were returned to a drop-box at each of the pre-schools and were collected by the researchers three weeks after recruitment commenced. Parents were eligible to participate if they: had a child aged 3-5 years who attended a participating pre-school; resided with that child at least four days per week; had some responsibility (i.e. at least half the time) in providing meals and snacks to their child; and were able to comprehend written and spoken English. Parents were ineligible if their child had dietary restrictions or allergies which were deemed by an independent Accredited Practising Dietitian to be in conflict with the Australian dietary predictor for fruit and vegetable consumption (e.g. fructose intolerance).

\section{Data collection}

Data were collected from parents by trained interviewers adhering to a survey script using a computer-assisted telephone interview. The survey took approximately $30 \mathrm{~min}$ to complete and was administered from April to October 2010. Parents were asked to answer with respect to their pre-school-aged child. If they had more than one child aged 3-5 years, they were instructed to answer all questions based on the child who would be having the next birthday. Parents were able to complete the telephone survey in a time that was convenient for them.

\section{Measures}

\section{Participant characteristics}

The survey included questions assessing the sociodemographic characteristics of both the parents and children. Parents reported their age, gender, highest education level, annual household income, and whether they identified as Indigenous, from fixed responses, as well as their child's age and gender. Items used to assess these demographics were sourced from the NSW Health Survey $^{(35)}$. Questions regarding number of days per week living in the same residence as the child and how often they were responsible for providing meals and snacks to the child were also included, to assess eligibility.

\section{Children's fruit and vegetable consumption}

Child consumption of fruits and vegetables was assessed via the fruit and vegetable subscale (eight items) from the Children's Dietary Questionnaire (CDQ) ${ }^{(11)}$. Parents were required to report the frequency and variety of fruits and vegetables their child had consumed: (i) in the past $7 \mathrm{~d}$; and (ii) in the last $24 \mathrm{~h}$. Frequency was assessed by asking how often the child ate fruits and/or vegetables (e.g. salad in sandwich and vegetables on the side, both eaten at lunch $=$ twice) within the past $24 \mathrm{~h}$ (nil, once, twice, 3 times, 4 times, $5+$ times) and the number of days within the past $7 \mathrm{~d}$ where the child had eaten fruit and/or vegetables (none, 1, 2, 3, 4, 5, 6, every day). To assess the variety of fruits and vegetables consumed, a list of twentyfive vegetables (including potato, but not hot chips) and nineteen fruits was read out to parents via telephone, and parents indicated which, if any, their child had consumed within: (i) the past $7 \mathrm{~d}$; and (ii) the previous $24 \mathrm{~h}$. An 'other' option was also provided to account for fruits or vegetables that were consumed but not already mentioned in the lists. The eight-item subscale was scored on a continuous scale from 0 to 28 , with a score of 14 or more representing meeting the national dietary guideline for fruit and vegetable consumption (based on dietary guidelines for children and adolescents from the Australian Guide to Healthy Eating) $)^{(11)}$. A higher score indicates greater consumption; an increase of 1 point could be equivalent to consuming an additional type of fruit or vegetable or consuming a fruit or vegetable at an additional occasion. Validity (Spearman's correlation coefficient $=0.58$ ) and reliability (intraclass correlation coefficient $=0.75$ ) of the fruit and vegetable subscale have been previously established in a sample of Australian children $^{(11)}$.

\section{Children's non-core food consumption}

Child consumption of non-core foods was assessed via the non-core foods subscale (twelve items) from the CDQ ${ }^{(11)}$. Parents were asked to report the frequency with which their child consumed each of twelve different categories of common non-core foods ('Takeaway, e.g. McDonalds, KFC, fish and chips/chicken shop', 'Lollies, muesli bars or fruit bars' and 'Chocolate - bar, block, coated biscuits') in the past $7 \mathrm{~d}$ (nil, once, twice, 3 times, 4 times, 5 times, 6+ times). The subscale is scored on a continuous scale from 0 to $10 \cdot 3$, with a score of $\geq 2$ established as exceeding the recommended amount of non-core foods based on the 
national dietary guidelines ${ }^{(11)}$. The higher the score, the greater the amount of non-core foods consumed. Validity (Spearman's correlation coefficient $=0 \cdot 31$ ) and reliability (intraclass correlation coefficient $=0.90$ ) of the subscale have also been established ${ }^{(11)}$.

\section{Time and cost of the provision of food}

Data were collected regarding the time (in minutes) that parents spent grocery shopping, the time (in minutes) spent preparing food for the household and the expenditure (in \$AU) on household groceries. Items were constructed by the research team for the purpose of the present study. Parents were required to report on: the average amount of time in minutes spent on grocery shopping for their household each week; the average amount of time in minutes spent preparing food for the household each day; and the approximate amount of money spent on food for the household (including takeaways and eating out) each week.

\section{Analysis}

Data were checked for normality and descriptive statistics were used to describe the sample. Parental education and annual household income were dichotomised based on cut-off points used in a previous study based on this data set ${ }^{(19)}$. CDQ scores were dichotomised into 'meeting dietary guidelines' and 'not meeting dietary guidelines' (i.e. fruit and vegetables subscale $<14$; non-core foods subscale $\geq 2$ ). Analysis was conducted using the statistical software program $\mathrm{JMP}^{\circledR}$ version 11.0 (SAS Institute Inc., 1989-2007). To investigate associations between diet and the provision of food variables, simple linear regression models were generated. The input variables were parental investment of time and cost for the provision of food, while the outcome variables were the CDQ subscale scores for (i) fruit and vegetable consumption and (ii) non-core food consumption. Given evidence suggesting associations between children's fruit and vegetable consumption and parental factors including income, education and the number of children (less than 16 years of age) in the household, the final multiple linear regression models were generated using the 'Fit Model' function with a personality of 'Standard Least Squares' and adjusted for these confounders ${ }^{(36-38)}$. Six models were generated comparing the two measures of parental investment of time (time shopping and time taken to prepare foods) and the measure of cost against the CDQ subscale scores for: (i) fruits and vegetables; and (ii) non-core foods. The data were cleaned and checked for implausible results, and such data were excluded from analysis. For time spent on food preparation each day, values greater than $240 \mathrm{~min}$ (i.e. $4 \mathrm{~h}$ spent preparing food every day) were regarded as implausible and excluded from analysis ( $n$ 4). A screening criterion of $P<0.05$ was determined as a significant association.

\section{Results}

Of the thirty-eight pre-schools contacted, seven preschools declined participation and one could not be contacted, with the thirty remaining pre-school supervisors consenting to participate. A total of 417 parents consented to the study from the thirty consenting preschools in the Hunter Region. Of the 417 parents who initially consented, ten refused to participate when contacted to complete the telephone survey, six did not meet the eligibility criteria and five could not be contacted, leaving 396 parents with completed survey data for analysis. The parent and child characteristics of the sample are described in Table 1. In total, 380 females (96\%) and sixteen males completed the survey, with a mean age of 35.45 (sD 5.35) years. A total of $47 \%$ of parents had completed a university degree. Seventeen parents refused to disclose or did not know their annual household income, while among the 379 parents who answered this question, $41 \%$ had an annual household income of \$AU 100000 or more. The child subject of the survey was female in $49 \%$ of cases and the mean age was 4.29 (SD 0.61) years. The mean number of children per household was 2.29 (SD 0.75). Among those surveyed, 2 and $3 \%$ of parents and children, respectively, identified as Aboriginal or Torres Strait Islander.

\section{Input variables}

The mean time parents spent each day preparing food for the household was 108 (SD 44.27) min (range 30-240 min). The median time spent preparing food was $120 \mathrm{~min}$. The mean time parents spent each week shopping for groceries for the household was 124 (SD 54.35) min (range 30$420 \mathrm{~min}$ ). The median time spent shopping for groceries

Table 1 Parent and child characteristics within the study sample; parents $(n 396)$ with a child aged $3-5$ years attending a pre-school participating in the Healthy Habits Trial, Hunter Region of New South Wales, Australia, April-October 2010 (baseline data)

\begin{tabular}{|c|c|c|}
\hline & $\begin{array}{l}n \text { or } \\
\text { Mean }\end{array}$ & $\%$ or SD \\
\hline \multicolumn{3}{|l|}{ Parent characteristics } \\
\hline Gender (female), $n$ and $\%$ & 380 & 96 \\
\hline Age (years), mean and SD & 35.45 & 5.35 \\
\hline $\begin{array}{l}\text { Aboriginal and/or Torres Strait Islander, } \\
n \text { and \% }\end{array}$ & 8 & 2 \\
\hline \multicolumn{3}{|l|}{ Highest education level, $n$ and $\%$} \\
\hline Completed TAFE/high school or less & 209 & 53 \\
\hline University & 187 & 47 \\
\hline \multicolumn{3}{|l|}{ Annual household income ${ }^{*}, n$ and $\%$} \\
\hline$<\$ A U 100000$ & 222 & 59 \\
\hline$\geq \$ A U 100000$ & 157 & 41 \\
\hline \multicolumn{3}{|l|}{ Child characteristics } \\
\hline Gender (female), $n$ and $\%$ & 193 & 49 \\
\hline Age (years), mean and SD & 4.29 & 0.61 \\
\hline $\begin{array}{l}\text { Aboriginal and/or Torres Strait Islander, } \\
n \text { and } \%\end{array}$ & 11 & 3 \\
\hline
\end{tabular}

${ }^{*} n 17$ excluded (don't know or refused). 
was $120 \mathrm{~min}$. The mean expenditure for food each week including takeaways and eating out was \$AU 238 (SD 80.91; range \$AU 80-700). There was no significant difference between male or female parents in reporting the time spent on food preparation $(P=0 \cdot 119)$, time spent on grocery shopping $(P=0 \cdot 167)$ or cost $(P=0 \cdot 190)$ spent.

\section{Outcome variables}

Children's fruit and vegetable consumption

The mean CDQ score for the fruit and vegetable subscale was 14.78 (sD 4.62 ; range $0-24$ ) out of a maximum score of 28. A total of $63 \%$ of children scored 14 or greater, indicating adherence to dietary guidelines for fruit and vegetable intake ${ }^{(11)}$.

\section{Children's non-core food consumption}

The mean CDQ score for the non-core foods subscale was $2 \cdot 53$ (SD 1.12; range $0 \cdot 29-5 \cdot 71$ ) out of a possible $10 \cdot 3$. A total of $64 \%$ of children had a score equal to or exceeding 2 , indicating they were exceeding dietary guidelines for non-core foods ${ }^{(11)}$.

The associations between children's dietary intakes and the parent time spent in provision and cost of food are displayed in Table 2. From the simple regression models, it was found that there were significant positive associations between the CDQ scores for non-core foods and parental investment of time for food preparation $(P=0.032)$ and cost of procurement $(P=0 \cdot 043)$. However, the magnitude of the effects was small (estimate $=0.003$ and 0.001, respectively). For fruit and vegetable consumption, there were no significant positive associations.
The multiple regression model included input variables (time spent preparing food for household per day, time spent procuring household groceries per week and cost of procurement per week), outcome variables and the potential confounders of parental education level, household income and number of children less than 16 years of age. The multiple regression model indicated there were significant positive associations between the CDQ scores for non-core foods and parental investment of time for food preparation (estimate $=0.003, \quad P=0.025$ ) and cost of procurement (estimate $=0.002, P=0.020$ ); however, the magnitude of the effect was small. Parent education level was found to be significantly associated with children's intake of fruits and vegetables when taken into consideration with time spent on food preparation $(P=0.013)$, time spent procuring food $(P=0.016)$ and cost of procurement $(P=0.027)$.

\section{Discussion}

The current study aimed to investigate the associations of parent-reported time and cost of the provision of food with the consumption of fruits and vegetables and noncore foods in a sample of Australian children of pre-school age. From the study sample of 396 Australian parents, it was found that there were no associations between the fruit and vegetable consumption of pre-schoolers and parental investment of time and cost spent on the provision of food. In addition, despite associations in other studies $^{(36,37)}$, household income and number of children were not significantly associated with pre-schoolers' fruit and vegetable consumption in this sample. However, similar to previous research, the study found that higher

Table 2 Associations between Children's Dietary Questionnaire subscale scores and parental investment of time and cost in the provision of food; parents ( $n$ 396) with a child aged 3-5 years attending a pre-school participating in the Healthy Habits Trial, Hunter Region of New South Wales, Australia, April-October 2010 (baseline data)

\begin{tabular}{|c|c|c|c|c|}
\hline & Estimate & SE & $r^{2}$ & $P$ value \\
\hline \multicolumn{5}{|l|}{ Simple regression model ( $n$ 392-395) } \\
\hline \multicolumn{5}{|l|}{ Fruit and vegetable subscale score } \\
\hline Time for food preparation per day (min) & 0.002 & 0.005 & $<0.001$ & 0.685 \\
\hline Time for grocery shopping per week (min) & 0.003 & 0.004 & 0.001 & 0.505 \\
\hline Total cost of food per week (\$AU) & -0.003 & 0.003 & 0.003 & 0.244 \\
\hline \multicolumn{5}{|l|}{ Non-core foods subscale score } \\
\hline Time for food preparation per day (min) & 0.003 & 0.001 & 0.012 & 0.032 \\
\hline Time for grocery shopping per week (min) & $<0.001$ & 0.001 & $<0.001$ & 0.799 \\
\hline Total cost of food per week ( $\$ A U)$ & 0.001 & $<0.001$ & 0.010 & 0.043 \\
\hline \multicolumn{5}{|l|}{ Multiple regression model ${ }^{*}(n$ 375-378) } \\
\hline \multicolumn{5}{|l|}{ Fruit and vegetable subscale score } \\
\hline Time for food preparation per day (min) & 0.003 & 0.005 & 0.028 & 0.631 \\
\hline Time for grocery shopping per week (min) & 0.003 & 0.004 & 0.026 & 0.519 \\
\hline Total cost of food per week (\$AU) & -0.003 & 0.003 & 0.026 & 0.275 \\
\hline \multicolumn{5}{|l|}{ Non-core foods subscale score } \\
\hline Time for food preparation per day (min) & 0.003 & 0.001 & 0.015 & 0.025 \\
\hline Time for grocery shopping per week (min) & $<0.001$ & 0.001 & 0.002 & 0.689 \\
\hline Total cost of food per week (\$AU) & 0.002 & $<0.001$ & 0.015 & 0.020 \\
\hline
\end{tabular}

Significant results are indicated in bold font.

${ }^{*}$ Adjusted for annual household income, parent education level and number of children less than 16 years of age in the household. 
parent education level was associated with higher fruit and vegetable consumption in pre-schoolers ${ }^{(36,37)}$. Regarding the second outcome variable, greater consumption of noncore foods was positively associated with the amount of time parents spend on food preparation and cost spent on procurement, although the magnitude of the effect was small. Therefore, our original study hypothesis that more time and cost spent on the provision of food would be associated with higher consumption of fruits and vegetables and lower consumption of non-core foods was not supported by the results.

In the current sample, it was identified that $64 \%$ of the pre-school children were reported to be consuming an excessive amount of non-core foods (exceeding the dietary guidelines), which is in accordance with previous reports of Australian pre-school children's consumption ${ }^{(15)}$. Comparatively, $63 \%$ of pre-school children in the sample were reported be consuming adequate amounts of fruits and vegetables to meet the dietary guidelines, which is substantially higher than the national average of $5 \cdot 1 \%^{(9)}$, indicating that the current sample may not have been representative in terms of fruit and vegetable consumption.

The mean cost spent on food each week in this sample ( $\$$ AU 238 per week) is comparable to the national average of \$AU 237 per week for household expenditure on foods and beverages ${ }^{(39)}$. Cost of household food in the present study included groceries, eating out and takeaways, and the study found that higher consumption of non-core foods was positively associated with cost of food procurement. The association might be attributed to more incidences of eating out and more money spent on noncore foods (i.e. fast foods ${ }^{(40)}$ ) compared with the money spent on fruits and vegetables ${ }^{(41)}$. It is recommended that future research explores the composition of total expenditure on food via tracking of receipts and that it investigates the contribution made by eating out, which may explain the higher cost associated with consumption of these foods. In addition, identifying more frequently consumed categories of non-core foods and their associated costs might help to better understand the cost breakdown (i.e. more money spent on sweetened beverages or on takeaways) and help to identify targets of specific categories of non-core foods for future interventions. Furthermore, surveying multiple parents and carers within a family, rather than one parent, may provide a more accurate estimate of total household expenditure on foods and beverages. This may in part explain why this relatively wealthy sample was spending only equivalent to the national average. Qualitative and quantitative research would also be of use to understand the reasons behind provision of non-core foods despite the high costs of these foods. For pre-school children from families of higher socio-economic status, there may be benefit in increased focus on interventions that aim to reduce consumption of non-core foods, rather than increasing consumption of fruits and vegetables.
In the current study, parental investment of time on food preparation was positively associated with consumption of non-core foods in pre-school children, although the magnitude was small. These results contradict findings in the adult population, whereby less time spent on food preparation was associated with more money spent on food procurement and eating out ${ }^{(30)}$. The high time investment reported by parents in the study might be attributable to the type of food cooked (i.e. slow cooking, stewing or baking requires more time compared with stir-frying or preparing simple foods that do not require cooking such as a sandwich). Hence, the lack of association between time spent on food preparation and consumption of fruits and vegetables may arise as some of these foods can be consumed whole with little or no preparation, and vegetables can be eaten raw, as salads or pre-packed and ready to use. Furthermore, some non-core foods that are often considered 'convenience foods' can still require a substantial amount of time to prepare prior to eating but require less effort (i.e. more 'passive' preparation) compared with the more 'active' preparation (e.g. chopping of vegetables and stir-frying) commonly required to prepare healthier foods. For example, a frozen pizza or meat pie would still be required to be cooked in the oven, following a period of pre-heating (e.g. $15 \mathrm{~min}$ pre-heating and $20 \mathrm{~min}$ cooking time, therefore total of $35 \mathrm{~min}$ spent). However, the time involved mostly consists of waiting time rather than active preparation. Since food preparation is a subjective term and could mean different things to different participants, further research is needed to explore time usage for food preparation in more detail; for example, the steps or cooking methods involved could greatly influence time reported in a survey. Clearer definitions of food preparation could be considered because cooking food in an oven, where nothing needs to be done, is a passive process but still could be counted by participants as 'preparing'; however, actively stir-frying and adding ingredients is more active and truer of food preparation. Another influence on the time spent preparing meals might be related to parent confidence and knowledge of cooking. For example, parents with more experience and familiarity with cooking would require less time to be spent on food preparation $^{(23,41-44)}$. Lack of cooking skills and food preparation knowledge may result in greater reliance on eating out or takeaway foods or buying of convenience meals ${ }^{(23,42)}$. Further research can quantitatively and qualitatively explore the reasons behind such barriers.

Previous studies have reported components of the food provision cycle (i.e. time and cost for acquisition, preparation and cooking of foods) as barriers to fruit and vegetable intake in young children. Findings from the present cross-sectional study, however, suggest that there were no associations between these barriers and consumption. Further exploration of such associations using validated measures are warranted to better inform the design of future parent-targeted interventions to improve child dietary intake. 
The present study is among the first to investigate these associations using multiple regression analysis with a measure of fruit and vegetable and non-core food consumption in children. There are several limitations to the study that have to be considered. First, the study is based on cross-sectional data and the findings cannot indicate causality. Second, due to the nature of the recruitment process (relying on supervisors to distribute study information and research assistants approaching a convenience subset of parents during the hours they attended the service), we cannot accurately report a recruitment rate and the sample may not be typical or representative of the population. In comparison to a random sample of preschool children's parents in the region, more parents are tertiary-level educated and have an annual household income above the regional average ${ }^{(45)}$ and a greater proportion of the children sampled meet or exceed dietary guidelines for the consumption of fruits and vegetables. Third, the data are based on parent report of the preschool child's consumption and hence parents might be over- or under-reporting their child's dietary intake. The CDQ used in the present study demonstrated low to moderate levels of accuracy; however, at the time of the study, it was the only tool with established reliability and validity in samples of Australian children that could be administered over the telephone. Fourth, there may be selection bias as parents more interested in nutrition/ health-related issues may be more likely to volunteer to the study. Furthermore, the validity and reliability of reporting time spent on food preparation, time spent on grocery shopping and cost spent on food are unknown as they were created specifically for the Healthy Habits Trial. Future studies should consider assessing the total number of adults and children in the household, so the total amount of money spent could be more meaningfully and accurately represented. The present study explored the combined consumption of fruits and vegetables and did not allow for separate analysis with parental investment of time and cost for the provision of food. Furthermore, collecting information from other parents and carers within the family who may also be involved in food preparation and shopping for food may find stronger associations. Future research should be conducted prospectively to investigate if the weak associations with non-core food consumption can be replicated across a time span, and then to determine if they are maintained across families from more diverse socio-economic backgrounds. It is recommended that future studies seek to address these limitations.

\section{Conclusion}

The present study found no associations between the fruit and vegetable consumption of children of pre-school age and the time and cost invested by their parents in preparing and procuring foods. It found that although noncore food consumption in pre-school children was associated with increased time and money spent on preparing and procuring food, the magnitude of the effect was small. As such, further investigation into the time spent in food preparation and the cost spent on food procurement and their associations with non-core foods in other samples of young children is warranted, as this could help address the increased consumption of non-core foods in pre-school children contributing to the overall public health issue of childhood obesity.

\section{Acknowledgements}

Acknowledgements: The authors would like to acknowledge the Healthy Habits Research Team and Advisory Group, the parents and children who participated in the study, and the telephone interviewers who collected the data. Financial support: The cluster-randomised controlled trial from which these data were collected was supported by the Cancer Institute New South Wales (grant number 08/ECF/1-18). Infrastructure support was provided by the Hunter Medical Research Institute and inkind support was provided by Hunter New England Population Health. Conflict of interest: None of the authors reported a conflict of interest. Authorship: S.X.J.L., R.W., T.B. and S.Y. conceived of the study; S.X.J.L. cleaned and analysed the data and led the writing of the manuscript. All authors contributed to drafts of the manuscript and read and approved the final version. Ethics of buman subject participation: This study was conducted according to the guidelines laid down in the Declaration of Helsinki and all procedures involving human subjects were approved by the Hunter New England Human Research Ethics Committee (reference number 08/10/15/5.09) and the University of Newcastle Human Research Ethics Committee. Written informed consent was obtained from all subjects.

\section{References}

1. Australian Institute of Health and Welfare (2014) Australia's Health 2014. Canberra: AIHW.

2. National Preventative Health Taskforce (2009) Australia: The Healthiest Country by 2020. http://www.health.gov.au/ internet/preventativehealth/publishing.nsf/content/E233 F8695823F16CCA2574DD00818E64/\$File/obesity-jul09.pdf (accessed April 2016).

3. World Health Organization (2012) Population-Based Approaches to Childhood Obesity Prevention. http:// www.who.int/dietphysicalactivity/childhood/WHO_new_ childhoodobesity_PREVENTION_27nov_HR_PRINT_OK.pdf (accessed April 2016).

4. Lim SS, Vos T, Flaxman AD et al. (2012) A comparative risk assessment of burden of disease and injury attributable to 67 risk factors and risk factor clusters in 21 regions, 1990-2010: 
a systematic analysis for the Global Burden of Disease Study 2010. Lancet 380, 2224-2260.

5. Hung HC, Joshipura KJ, Jiang R et al. (2004) Fruit and vegetable intake and risk of major chronic disease. J Natl Cancer Inst 96, 1577-1584.

6. Mazarello Paes V, Ong KK \& Lakshman R (2015) Factors influencing obesogenic dietary intake in young children $(0$ 6 years): systematic review of qualitative evidence. BMJ Open 5, e007396.

7. Krølner R, Rasmussen M, Brug J et al. (2011) Determinants of fruit and vegetable consumption among children and adolescents: a review of the literature. Part II: qualitative studies. Int J Behav Nutr Phys Act 8, 112.

8. National Health and Medical Research Council (2013) Australian Dietary Guidelines Summary. Canberra: NHMRC.

9. Australian Bureau of Statistics (2015) National Health Survey: First Results, 2014-15. http://www.abs.gov.au/ausstats/ abs@.nsf/Lookup/by\%20Subject/4364.0.55.001 2014-15 Main $\% 20$ Features $\sim$ Children's\%20risk\%20factors 31 (accessed April 2016).

10. NSW Ministry of Health (2013) Eat More Fruit and Vegies. https://www.healthykids.nsw.gov.au/downloads/file/textand linkbasedsidepanelteaser/eatmorefruitandveg.pdf (accessed May 2018).

11. Magarey A, Golley R, Spurrier N et al. (2009) Reliability and validity of the Children's Dietary Questionnaire; a new tool to measure children's dietary patterns. Int J Pediatr Obes $\mathbf{4}$, 257-265.

12. Craigie AM, Lake AA, Kelly SA et al. (2011) Tracking of obesity-related behaviours from childhood to adulthood: a systematic review. Maturitas 70, 266-284.

13. Queensland Health (2003) Primary Prevention of Chronic Diseases: The Role of Nutrition. https://www.health.qld.gov. $\mathrm{au} / \mathrm{ph} /$ Documents/hpu/19345.pdf (accessed April 2016).

14. Duncanson K, Burrows T \& Collins C (2013) Effect of a lowintensity parent-focused nutrition intervention on dietary intake of 2- to 5-year olds. J Pediatr Gastroenterol Nutr 57, 728-734.

15. Rangan AM, Randall D, Hector DJ et al. (2008) Consumption of 'extra' foods by Australian children: types, quantities and contribution to energy and nutrient intakes. Eur J Clin Nutr 62, 356-364.

16. Hall L, Collins CE, Morgan PJ et al. (2011) Children's intake of fruit and selected energy-dense nutrient-poor foods is associated with fathers' intake. J Am Diet Assoc 111, 1039-1044.

17. National Health and Medical Research Council (2013) Clinical Practice Guidelines for the Management of Overweight and Obesity in Adults, Adolescents and Children in Australia - Systematic Review. Melbourne: NHMRC.

18. Vaughn AE, Tabak RG, Bryant MJ et al. (2013) Measuring parent food practices: a systematic review of existing measures and examination of instruments. Int J Behav Nutr Phys Act 10, 61.

19. Wyse RJ, Campbell E, Nathan N et al. (2011) Associations between characteristics of the home food environment and fruit and vegetable intake in preschool children: a crosssectional study. BMC Public Health 11, 938.

20. Spurrier NJ, Magarey AA, Golley R et al. (2008) Relationships between the home environment and physical activity and dietary patterns of preschool children: a crosssectional study. Int J Behav Nutr Phys Act 5, 31.

21. Bava CM, Jaeger SR \& Park J (2008) Constraints upon food provisioning practices in 'busy' women's lives: trade-offs which demand convenience. Appetite 50, 486-498.

22. Ling J, B Robbins L \& Hines-Martin V (2015) Perceived parental barriers to and strategies for supporting physical activity and healthy eating among head start children. J Community Health 41, 593-602.
23. Soliah LAL, Walter JM \& Jones SA (2012) Benefits and barriers to healthful eating: what are the consequences of decreased food preparation ability? Am J Lifestyle Med $\mathbf{6}$, $152-158$

24. Carroll JD, Demment MM, Stiles SB et al. (2011) Overcoming barriers to vegetable consumption by preschool children: a child care center buying club. J Hunger Environ Nutr 6, 153-165.

25. Dwyer J, Needham L, Simpson JR et al. (2008) Parents report intrapersonal, interpersonal, and environmental barriers to supporting healthy eating and physical activity among their preschoolers. Appl Physiol Nutr Metab 33, 338-346.

26. Mushi-Brunt C, Haire-Joshu D \& Elliott M (2007) Food spending behaviors and perceptions are associated with fruit and vegetable intake among parents and their preadolescent children. J Nutr Educ Behav 39, 26-30.

27. Cafer AM \& Kaiser ML (2016) An analysis of differences in predictors of food affordability between rural and urban counties. J Poverty 20, 34-55.

28. Australian Bureau of Statistics (2009) Trends in Household Work. Canberra: ABS.

29. Produce for Better Health Foundation (2014) Primary Shoppers' Attitudes and Beliefs Related to Fruit and Vegetable Consumption, 2012 vs 2014. http://pbhfoundation.org/pdfs/ about/res/pbh_res/PrimaryShopperReport_WEB.pdf (accessed May 2018).

30. Monsivais P, Aggarwal A \& Drewnowski A (2014) Time spent on home food preparation and indicators of healthy eating. Am J Prev Med 47, 796-802.

31. Larson NI, Perry CL, Story M et al. (2006) Food preparation by young adults is associated with better diet quality. $J \mathrm{Am}$ Diet Assoc 106, 2001-2007.

32. McLaughlin C, Tarasuk V \& Kreiger N (2003) An examination of at-home food preparation activity among lowincome, food-insecure women. J Am Diet Assoc 103, $1506-1512$.

33. Wyse RJ, Wolfenden L, Campbell E et al. (2010) A cluster randomised trial of a telephone-based intervention for parents to increase fruit and vegetable consumption in their 3- to 5-year-old children: study protocol. BMC Public Health 10, 216.

34. Wyse R, Wolfenden L, Campbell E et al. (2012) A cluster randomized controlled trial of a telephone-based parent intervention to increase preschoolers' fruit and vegetable consumption. Am J Clin Nutr 96, 102-110.

35. Centre for Epidemiology and Research (2009) 2008 Summary Report on Adult Health from the New South Wales Population Health Survey. Sydney: Department of Health.

36. Sausenthaler S, Kompauer I, Mielck A et al. (2007) Impact of parental education and income inequality on children's food intake. Public Health Nutr 10, 24-33.

37. Cooke LJ, Wardle J, Gibson EL et al. (2004) Demographic, familial and trait predictors of fruit and vegetable consumption by pre-school children. Public Health Nutr $\mathbf{7}$, 295-302.

38. Australian Bureau of Statistics (2013) Spending Patterns of Couple Families. Canberra: ABS.

39. Australian Institute of Health and Welfare (2012) Australia's Food \& Nutrition 2012. Canberra: AIHW.

40. Australian Bureau of Statistics (2011) Household Expenditure Survey, Australia: Summary of Results, 2009-10. Canberra: ABS.

41. Australian Bureau of Statistics (2012) In Pursuit of $2 \& 5-$ Fruit and Vegetable Consumption in Australia. Canberra: ABS.

42. Reicks M, Trofholz AC, Stang JS et al. (2014) Impact of cooking and home food preparation interventions among 
adults: outcomes and implications for future programs. J Nutr Educ Behav 46, 259-276.

43. Swanson V, Power KG, Crombie IK et al. (2011) Maternal feeding behaviour and young children's dietary quality: a cross-sectional study of socially disadvantaged mothers of two-year old children using the Theory of Planned Behaviour. Int J Behav Nutr Phys Act 8, 65.
44. Winkler E \& Turrell G (2010) Confidence to cook vegetables and the buying habits of Australian households. J Am Diet Assoc 110, 5 Suppl., S52-S61.

45. Wolfenden L, Hardy LL, Milat A et al. (2011) Prevalence and socio-demographic associations of overweight and obesity among children attending child-care services in rural and regional New South Wales Australia. Nutr Diet 68, 15-20. 